\title{
Miliary Tuberculosis Associated with Chronic Neutrophilic Leukemia
}

\author{
Keishi Sugino ${ }^{1}$, Kyoko Gocho ${ }^{1}$, Hiroki Ota ${ }^{1}$, Minaho Kobayashi ${ }^{1}$, Go Sano ${ }^{1}$, \\ Kazutoshi Isobe ${ }^{1}$, Yujiro Takai ${ }^{1}$, Haruka Izumi ${ }^{2}$, Yasunobu Kuraishi ${ }^{2}$, Kazutoshi Shibuya ${ }^{3}$ \\ and Sakae Homma ${ }^{1}$
}

\begin{abstract}
We report a case of miliary tuberculosis associated with chronic neutrophilic leukemia (CNL). A 70-yearold woman was referred to our hospital complaining of a 1-month history of persistent fever and anorexia. Chest and abdominal computed tomography images revealed diffuse small nodular lesions in the bilateral lung fields and extreme splenomegaly. Sputum cultures isolated Mycobacterium tuberculosis. After antituberculous therapy for 1 year, the patient underwent splenectomy for massive splenomegaly and progressive leukocytosis. The presence of the homozygous JAK2 V617F tyrosine kinase mutation was also demonstrated in the peripheral blood. She was finally diagnosed as having miliary tuberculosis associated with CNL based on the histopathological examination of spleen. The patient was treated with a daily dose of $500 \mathrm{mg}$ of hydroxyurea. As a result, 18 months after the splenectomy, her leukocyte count was decreased and her clinical condition was markedly improved; there was no relapse of the CNL.
\end{abstract}

Key words: chronic neutrophilic leukemia, miliary tuberculosis, splenomegaly

(Inter Med 48: 1283-1287, 2009)

(DOI: 10.2169/internalmedicine.48.2080)

\section{Introduction}

Chronic neutrophilic leukemia (CNL) is a rare myeloproliferative disease, characterized by sustained peripheral blood neutrophilia, an increase in serum vitamin B12 and neutrophil alkaline phosphatase (NAP), an absence of Philadelphia chromosome or BCR/ABL fusion, and hepatosplenomegaly (1). In contrast, splenic tuberculosis is usually seen in patients with disseminated miliary tuberculosis, which is reported in up to $80-100 \%$ of such patients at autopsy (2). However, little has been reported on miliary tuberculosis associated with CNL. We report a very rare case of miliary tuberculosis associated with CNL which was confirmed by histopathological examination of the resected spleen and the detection of the homozygous JAK2 V617F tyrosine kinase mutation.

\section{Case Report}

A 70-year-old woman was referred to our hospital with a 1-month history of persistent fever and anorexia. In addition, she had been aware of an abdominal mass for 6 months prior to her admission. She had no history of alcohol problems or smoking. Physical findings on admission were: body temperature, $38.5^{\circ} \mathrm{C}$; heart rate, 98 beats $/ \mathrm{min}$ with regular rhythm; respiratory rate, 18 breaths/min; and blood pressure, 116/78 $\mathrm{mmHg}$. Chest auscultation showed coarse crackles in the right lung. Her spleen was palpable over a width of $12 \mathrm{~cm}$ below the costal margin. Laboratory data showed a leukocyte count of $37,400 / \mu \mathrm{L}$ with $88 \%$ neutrophils, C-reactive protein of $1.3 \mathrm{mg} / \mathrm{dL}$, and an erythrocyte sedimentation rate of $68 \mathrm{~mm} /$ hour. Arterial blood gas analysis revealed $\mathrm{PaO}_{2}$ of 66.0 torr and $\mathrm{PaCO}_{2}$ of 35.6 torr on room air. Chest X-ray obtained on admission showed infil-

${ }^{1}$ Division of Respiratory Medicine, Toho University Omori Medical Center, Tokyo, ${ }^{2}$ Division of Hematology and Oncology, Toho University Omori Medical Center, Tokyo and ${ }^{3}$ Division of Pathology, Toho University Omori Medical Center, Tokyo

Received for publication January 20, 2009; Accepted for publication April 8, 2009

Correspondence to Dr. Keishi Sugino, keishi_sugino@ybb.ne.jp 


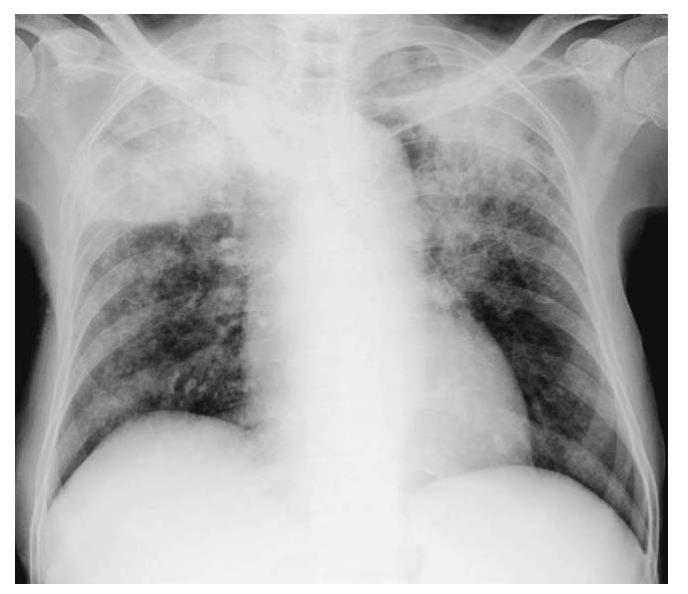

Figure 1. Chest X-ray obtained on admission showed infiltrative shadows with an airbronchogram in the bilateral upper lung fields with diffuse multiple small nodular shadows.
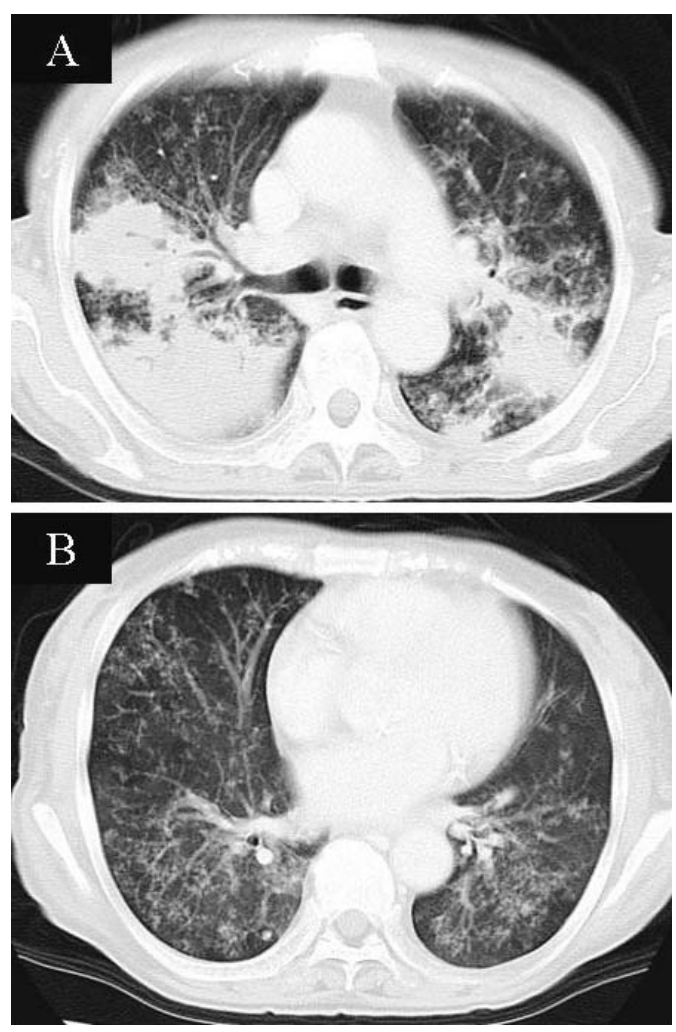

Figure 2. Chest CT on admission, showing: A) consolidation with an airbronchogram in the bilateral upper lobes. B) tree-in-bud appearance and multiple small nodular lesions in the bilateral lower lobes.

trative shadows with an airbronchogram in the bilateral upper lung fields and multiple small nodular shadows in the bilateral lung fields (Fig. 1). Chest computed tomography (CT) revealed consolidation with an air bronchogram in the bilateral upper lobes and tree-in-bud appearance and multiple small nodular lesions in the bilateral lower lobes (Fig. 2). Abdominal CT showed massive splenomegaly measuring $20 \times 16 \times 7 \mathrm{~cm}$ in size with multiple low density areas (Fig. 3). Sputum cultures isolated Mycobacterium tuber-

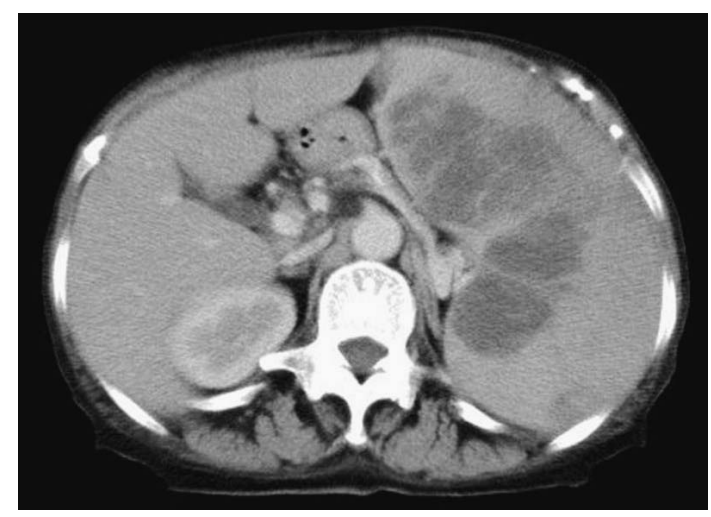

Figure 3. Abdominal CT showed splenomegaly (dimensions, $20 \times 16 \times 7 \mathrm{~cm}$ ) with multiple low density areas.

culosis, but without other common bacteria or fungi. Serum human immunodeficiency virus anitibody test was negative. The tuberculous polymerase chain reaction of sputum was positive. The patient was diagnosed as having miliary tuberculosis complicated by splenomegaly and a leukemoid reaction. Although miliary tuberculosis was improved by combination therapy with anti-tuberculous agents including isoniazid (INH, $300 \mathrm{mg}$ once daily) and rifampicin (RFP, $450 \mathrm{mg}$ once daily) for 1 year, together with ethambutol (EB, 750 $\mathrm{mg}$ once daily) for the initial 6 months and pyrazinamide (PZA, 1,200 mg twice daily) for the initial 2 months, the splenomegaly remained unchanged and leukocytosis progressed. Therefore, the patient underwent bone marrow aspiration with biopsy and a splenectomy in order to arrive at a correct diagnosis and to determine the correct treatment for her splenomegaly and leukocytosis. The differentiation of the bone marrow aspirate showed the following values: blast, $0.8 \%$; promyelocytes, $3.8 \%$; myelocytes, $12.4 \%$; metamyelocytes, $18.4 \%$; band neutrophils, $13.2 \%$; segmented neutrophils, 33.4\%; monocytes, $1.0 \%$; lymphocytes, $7.4 \%$; plasma cells, less than 1\%; eosinophils, $0.2 \%$; basophils, $1.2 \%$; erythrocytes, $8.2 \%$, respectively. Bone marrow aspirate and peripheral blood smear showed proliferation of hypersegmented neutrophils (Fig. 4A, B). The bone marrow biopsy specimen showed hypercellularity with marked granulocytic proliferation which mainly comprised stabforms and segmented neutrophils and a myeloid-to-erythroid (M:E) ratio of 5:1 (Fig. 5). A sample from her surgically-resected enlarged spleen, which weighed approximately $1,300 \mathrm{~g}$ showed multiple whitish nodules up to $4 \times 4.5 \times 3 \mathrm{~cm}$ in size (Fig. 6). Histopathological examination of the spleen showed an increased number of neutrophils at various stages of maturation (Fig. 7A) and multiple epithelioid cell granulomas with central caseous necrosis (Fig. 7B). Hematological examination showed leukocytosis of $80,500 / \mu \mathrm{L}$ with $70 \%$ neutrophils and an increased neutrophil alkaline phosphatase score of 401. The serum level of granulocyte colony stimulating factor was less than $10 \mathrm{pg} / \mathrm{mL}$, and that of vitamin B12 was $990 \mathrm{pg} / \mathrm{mL}$ (normal range, 180-914 pg/mL). Chromosome analysis revealed a normal karyotype (ie, 46, 


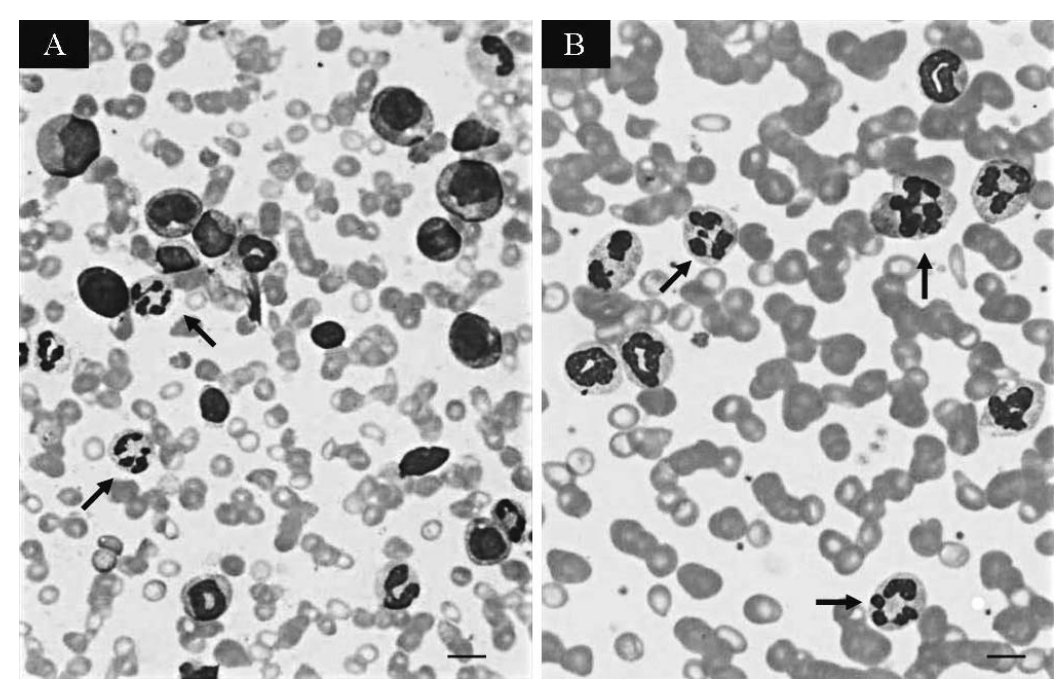

Figure 4. A) Bone marrow aspirate and B) peripheral blood smear. In both images, increased hypersegmented neutrophils are indicated by arrows (May-Grunwald Giemsa stain) (scale bar $=50 \mu \mathrm{m})$.

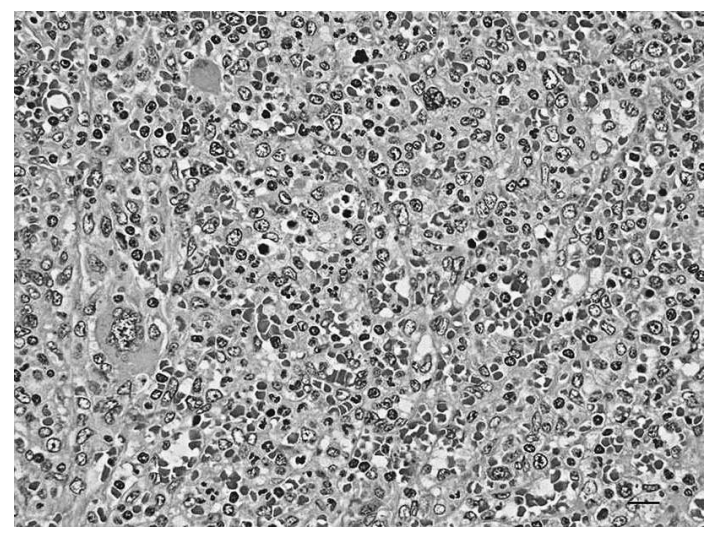

Figure 5. The bone marrow biopsied specimen showed hypercellularity with marked granulocytic proliferation, mainly comprising stabforms and segmented neutrophils with a myeloid-to-erythroid (M:E) ratio of 5:1 (Hematoxylin and Eosin staining) (scale bar $=150 \mu \mathrm{m})$.

$\mathrm{XX}$ ). Fluorescent in situ hybridization (FISH) analysis was negative for a BCR/ABL translocation. Furthermore, the presence of the homozygous JAK2 V617F tyrosine kinase mutation was demonstrated in the peripheral blood. According to these findings, the patient was diagnosed as having miliary tuberculosis associated with CNL. Because it took a long time to reach the definite diagnosis of CNL histopathologically, oral hydroxyurea was started at a daily dose of $500 \mathrm{mg}$ three months after splenectomy and was continued for 18 months. Her leukocyte count subsequently decreased to between 12,000 and $20,000 / \mu \mathrm{L}$, and her clinical condition markedly improved; 18 months post-splenectomy there was no relapse of CNL (Fig. 8).

\section{Discussion}

CNL is an extremely rare myeloproliferative disorder, and

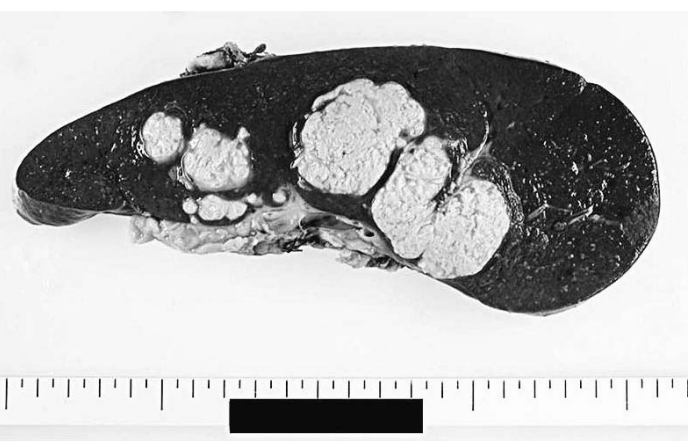

Figure 6. Macroscopy of the spleen revealed multiple whitish nodules up to $4 \times 4.5 \times 3 \mathrm{~cm}$ in size (scale bar: 1 division= 0.5 cm).

was first described by Tuohy in 1920 (3). The diagnosis of CNL is mainly based on the following criteria: (i) persistent mature neutrophilia in the peripheral blood; (ii) hypercellular bone marrow with increased neutrophilic granulocytes; (iii) hepatosplenomegaly; (iv) the absence of BCR/ABL fusion gene (1). The present case met all these diagnostic criteria. In addition however, a diagnosis should be confirmed by excluding other diseases which may cause a leukemoid reaction such as malignancy, severe infection (sepsis, miliary tuberculosis), toxication or acute hemolysis (4). In the present case, there was no evidence of an underlying malignancy, toxication or acute hemolysis. Although miliary tuberculosis was confirmed by sputum culture and histopathological findings of the resected spleen, based on her clinical course, the patient was not diagnosed as having a leukemoid reaction. Rather, she was diagnosed with progressive leukocytosis and splenomegaly in spite of an effective treatment for miliary tuberculosis. Consequently, the patient was diagnosed as having CNL, with miliary tuberculosis and splenomegaly, but no leukemoid reaction. CNL usually occurs in patients, of either sex, aged over 50 years. 

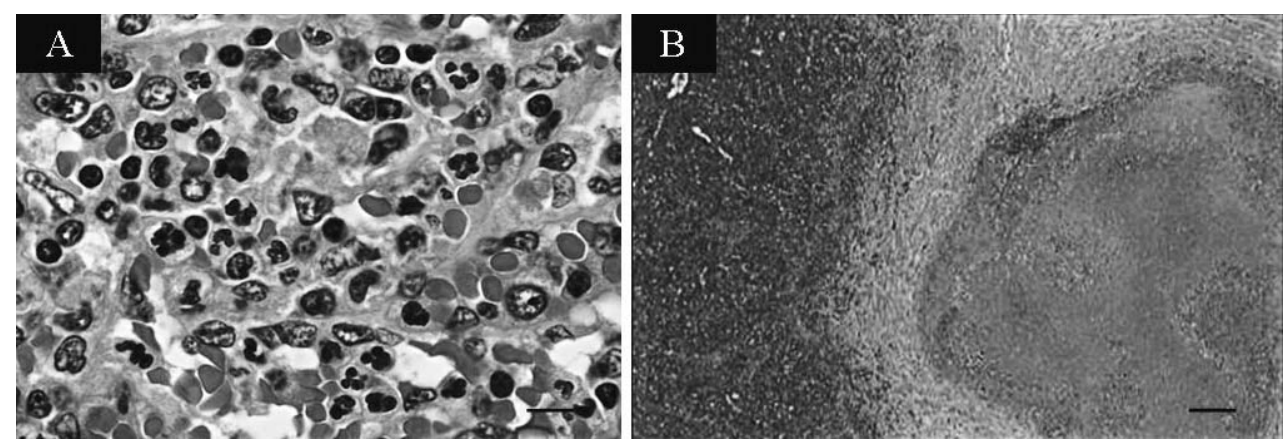

Figure 7. Microscopic findings of the spleen. A) An increase in neutrophils at various stages of maturation is apparent (Hematoxylin and Eosin staining) (scale bar=50 $\mu \mathrm{m}$ ). B) Multiple epithelioid cell granulomas with central caseous necrosis are apparent (Hematoxylin and Eosin staining) (scale bar $=\mathbf{3 0 0} \mu \mathrm{m}$ ).

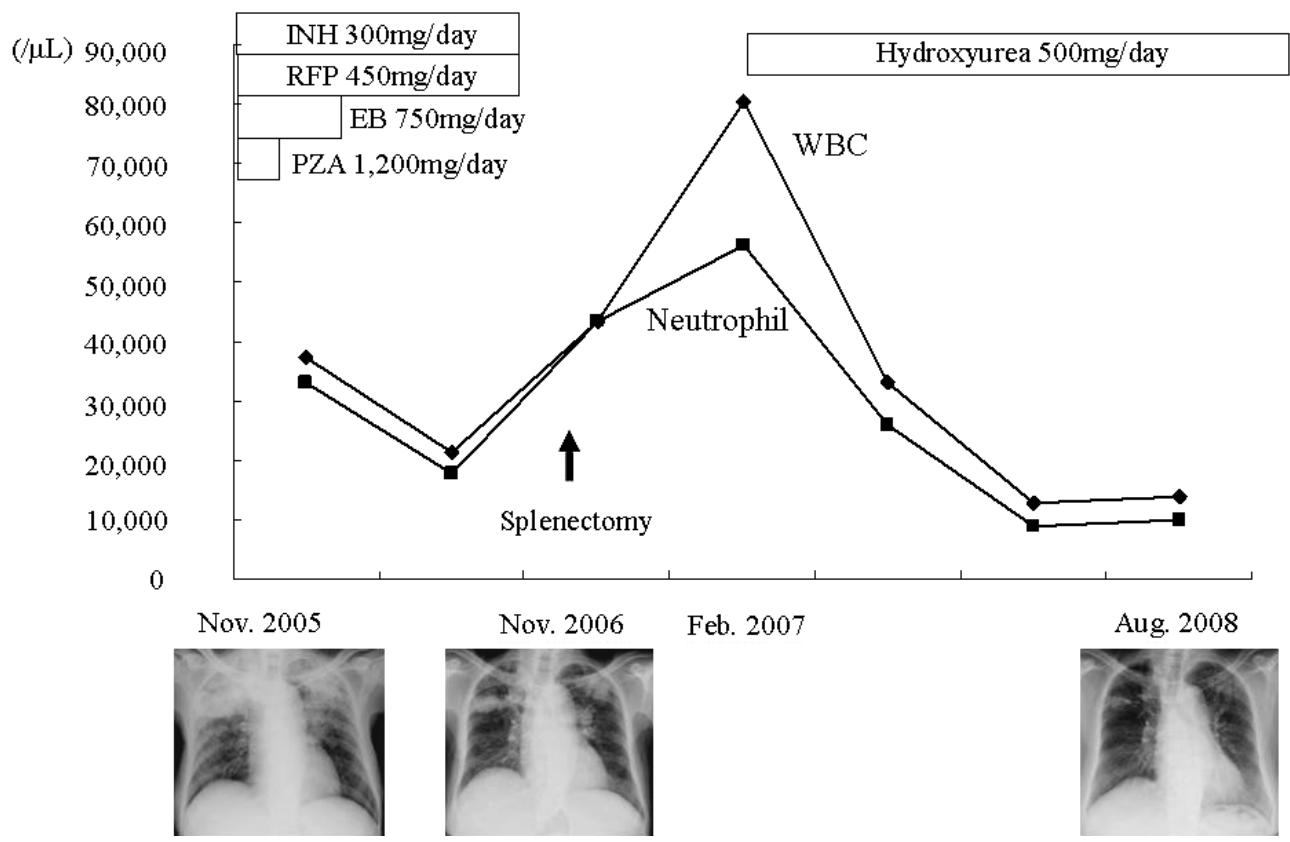

Figure 8. Clinical course of the present case.

Splenomegaly is the main clinical feature, and it may be progressive and symptomatic (5). In the present case, the appearance of a bloating sensation in the abdomen due to splenomegaly led to the diagnosis of the disease. Recently, a point mutation in DNA coding Jak homology domain 2 pseudokinase domain of JAK2 has been found in a significant proportion of patients with classical myeloproliferative disease and chronic idiopathic myelofibrosis $(6,7)$. Several papers reported the presence of the JAK2 V617F mutation in CNL patients (8-10). The detection of this genetic mutation in our patient also strongly supported the association of CNL.

Splenic involvement in tuberculosis is usually associated with dissemination of advanced pulmonary or miliary tuberculosis (11). Batra et al (12) reported that the incidence of splenic tuberculosis was approximately $8 \%$. In contrast, Thoeni and Margulis described that the incidence of hepatosplenic involvement of tuberculosis was as high as 80-
$100 \%$ in autopsied cases with disseminated pulmonary tuberculosis. On abdominal CT, splenic lesions of tuberculosis typically appear as multiple, rounded and hypodense, and between 1-2 $\mathrm{cm}$ in diameter (13). But, these findings are non-specific as they are also seen in abscesses or lymphomas. Indeed, it is difficult to differentiate splenic tuberculosis from these diseases by CT or other images. Therefore, a definite diagnosis of splenic tuberculosis may still require surgical intervention. In the present case, although combination therapy with INH, RFP, EB and PZA was given for 1 year, the patient developed persistent splenomegaly and leukocytosis. Therefore, it was decided to perform a splenectomy, which showed the coexistence of CNL and tuberculosis in the resected spleen. It has been known that long-term corticosteroid administration, diabetes mellitus, acquired immunodeficiency syndrome and hematological malignancy impair the function of neutrophils and/or macrophages which play an important role in preventing the development 
of active Mycobacterium tuberculosis. The functional characteristics of neutrophils in CNL patients in particular have been studied in some detail. Dotten et al (14) reported that bactericidal activity is reduced and levels of lysozyme and beta-glucuronidase are decreased, although phagocytic function is normal. In contrast, Zittoun et al (5) reported normal neutrophil function in two CNL cases. Moreover, it has been suggested that neutrophil functions, such as superoxide production, nitroblue tetrazolium reduction activity and phagocytosis are elevated (15). These different results for neutrophil functions with CNL may be due to differences in the pathophysiology of each case or to technical problems in the assessment of neutrophil functions. On the other hand, deletion of the long arm of chromosome 20 is a common chromosomal abnormality in CNL (16), so that a variety of chromosomal aberrations may allow for some alteration in CNL neutrophil function. Therefore, we can not account for the cause of miliary tuberculosis with any certainty, and it may merely occur accidentally.

Experience in treating CNL is limited because of the rarity of the disease. Optimal therapy is unclear and is mainly aimed at symptomatic support. Oral chemotherapeutic agents such as busulphan and hydroxyurea are useful in controlling the high leukocyte counts, but are not curative (17). Splenectomy or splenic irradiation has been used and found to be effective in reducing symptomatic splenomegaly although this may lead to the degeneration of neutrophils (18). The present case was treated with a daily dose of $500 \mathrm{mg}$ hydroxyurea. As a result, 18 months following the splenectomy, her leukocyte count had decreased and her clinical condition was markedly improved; there had been no relapse of CNL.

To our knowledge, although a few papers have already reported the association of CNL and mycobacterium infection such as pulmonary mycobacterium kansasii infection and meningeal tuberculosis $(19,20)$, this is an extremely rare reported case of miliary tuberculosis associated with CNL which was confirmed by histopathological examination of the resected spleen. In cases of unexplained leukocytosis, CNL should be included in the differential diagnosis.

\section{Acknowledgement}

We are grateful to T. Nikaido for the advice and analysis of the patient's pathology (The Department of Pathology, Jikei University School of Medicine, Tokyo, Japan).

\section{References}

1. Imbert M, Bain B, Pierre R, Vardiman JW, Brunning RD, Flandrin G. Chronic neutrophilic leukaemia. In: World Health Organization Classification of Tumours: Tumours of Haematopoietic and Lymphoid Tissues. IARC Press, Lyon, France, 2001: 27-28.

2. Thoeni RF, Margulis AR. Gastrointestinal tuberculosis. Semin Roentgenol 14: 283-294, 1979.

3. Tuohy EA. A case of splenomegaly with polymorphonuclear neutrophil hyperleukocytosis. Am J Med Sci 160: 18-25, 1920.

4. Sakka V, Tsiodras S, Giamarellos-Bourboulis EJ, Giamarellou H. An update on the etiology and diagnostic evaluation of a leukemoid reaction. Eur J Intern Med 17: 394-398, 2006.

5. Zittoun R, Réa D, Hoang Ngoc L, Ramond S. Chronic neutrophilic leukemia: A study of four cases. Ann Hematol 68: 55-60, 1994.

6. Baxter EJ, Scott LM, Campbell PJ, et al. Acquired mutation of the tyrosine kinase JAK2 in human myeloproliferative disorders. Lancet 365: 1054-1061, 2005.

7. Vizmanos JL, Ormazabal C, Larrayoz MJ, Cross NC, Calasanz MJ. JAK2 V617F mutation in classic chronic myeloproliferative disease: a report on a series of 349 patients. Leukemia 20: 534535, 2006.

8. Mc Lornan DP, Percy MJ, Jones AV, Cross NC, Mc Mullin MF. Chronic neutrophilic leukemia with an associated V617F JAK2 tyrosine kinase mutation. Heamatologica 90: 1696-1697, 2005.

9. Lea NC, Lim Z, Westwood NB, et al. Presence of JAK2 V617F tyrosine kinase mutation as a myeloid-lineage-specific mutation in chronic neutrophilic leukaemia. Leukemia 20: 1324-1326, 2006.

10. Kako S, Kanda Y, Sato T, et al. Early relapse of JAK2 V617F positive chronic neutrophilic leukemia with central nervous system infiltration after unrelated bone marrow transplantation. Am J He- matol 82: 386-390, 2007.

11. Weir MR, Thornton GF. Extrapulmonary tuberculosis: Experience of a community hospital and review of the literature. Am J Med 79: 467-478, 1985.

12. Batra A, Sarma D, Paul SB. Sonographic appearances in abdominal tuberculosis. J Clin Ultrasound 28: 233-245, 2000.

13. Gulati MS, Sarma D, Paul SB. CT appearances in abdominal tuberculosis. Clin Imaging 23: 51-59, 1999.

14. Dotten DA, Pruzanski W, Wong D. Functional characterization of the cells in chronic neutrophilic leukemia. Am J Hematol 12: 157$165,1982$.

15. Ohtsuki T, Katsura Y, Mizukami H, et al. Elevated neutrophil function in chronic neutrophilic leukemia. Am J Hematol 41: 5056, 1992.

16. Frank MB, Norwood TH, Willerford DM. Chimeric del20q in a case of chronic neutrophilic leukemia. Am J Hematol 64: 229-231, 2000.

17. Gan GG, Pasagna JJF, Eow GI, Nadarajan VS. Chronic neutrophilic leukaemia. Singapore Med J 48: 74-76, 2007.

18. Reilly JT. Chronic neutrophilic leukaemia; a distinct clinical entity? Br J Haematol 116: 10-18, 2002.

19. Okada S, Miyoshi Y, Takizawa Y, et al. Neutrophil dysfunction in chronic neutrophilic leukemia without rearrangements of bcr and immunoglobulin heavy chain genes. Rinsho Ketsueki 30: 18811885, 1989 (in Japanese).

20. Delgado BP, Rubio-Felix D, Garcia Zueco JC, Capablo JL, Alvarez R. Chronic neutrophilic leukemia and meningeal tuberculosis: chance or cause? Sangre (Barc) 40: 235-237, 1995.

(C) 2009 The Japanese Society of Internal Medicine http://www.naika.or.jp/imindex.html 